\title{
Using national registry data and record linkage to inform post-market surveillance of prosthetic aortic valve models over 15-years
}

DOI:

10.1001/jamainternmed.2016.6936

\section{Document Version \\ Accepted author manuscript}

Link to publication record in Manchester Research Explorer

Citation for published version (APA):

Hickey, G. L., Bridgewater, B., Grant, S. W., Deanfield, J., Parkinson, J., Bryan, A. J., Dalrymple-Hay, M., Moat, N., Buchan, I., \& Dunning, J. (2017). Using national registry data and record linkage to inform post-market surveillance of prosthetic aortic valve models over 15-years. JAMA Internal Medicine, 177(1), 79-86.

https://doi.org/10.1001/jamainternmed.2016.6936

\section{Published in:}

JAMA Internal Medicine

\section{Citing this paper}

Please note that where the full-text provided on Manchester Research Explorer is the Author Accepted Manuscript or Proof version this may differ from the final Published version. If citing, it is advised that you check and use the publisher's definitive version.

\section{General rights}

Copyright and moral rights for the publications made accessible in the Research Explorer are retained by the authors and/or other copyright owners and it is a condition of accessing publications that users recognise and abide by the legal requirements associated with these rights.

\section{Takedown policy}

If you believe that this document breaches copyright please refer to the University of Manchester's Takedown Procedures [http://man.ac.uk/04Y6Bo] or contact uml.scholarlycommunications@manchester.ac.uk providing relevant details, so we can investigate your claim.

\section{OPEN ACCESS}




\section{Using national registry data and record linkage to inform post-market}

2 surveillance of prosthetic aortic valve models over 15-years

Graeme L. Hickey ${ }^{1,2}$ [Ph.D.], Ben Bridgewater ${ }^{2,3}$ [Ph.D., FRCS (C-Th)], Stuart W. Grant ${ }^{2,4}$ [MB

4 ChB, Ph.D.], John Deanfield ${ }^{2}$ [MB BChir, FRCP], John Parkinson ${ }^{2}$ [Ph.D.], Alan J. Bryan ${ }^{5}$ [MD, FRCS (C-Th)], Malcolm Dalrymple-Hay ${ }^{6}$ [Ph.D., FRCS (C-Th)], Neil Moat ${ }^{7}$ [FRCS], Iain

6 Buchan $^{8}$ [MD, FFPH], Joel Dunning ${ }^{9}$ [Ph.D., FRCS (C-Th)]

$8{ }^{1}$ University of Liverpool, Department of Biostatistics, Waterhouse Building (Block F), 1-5 Brownlow Street, Liverpool, L69 3GL, UK

$10{ }^{2}$ University College London, National Institute for Cardiovascular Outcomes Research (NICOR), 1 St Martin le Grande, London, EC1A 4NP, UK

$12{ }^{3}$ Computer Science Corporation, One Pancras Square, Kings Cross, London, N1C 4AG, UK

${ }^{4}$ Department of Cardiothoracic Surgery, Lancashire Cardiac Centre, Blackpool Victoria

14 Hospital, Whinney Heys Road, Blackpool, FY3 8NR, UK

${ }^{5}$ Department of Cardiac Surgery, Bristol Heart Institute, Bristol Royal Infirmary, Bristol, BS2

$168 \mathrm{HW}, \mathrm{UK}$

${ }^{6}$ South West Cardiothoracic Centre, Derriford Hospital, Derriford, Plymouth, PL68DH, UK

$18{ }^{7}$ Cardiovascular Biomedical Research Unit, Royal Brompton Hospital, Sydney Street, London SW3 6NP, UK 
$20{ }^{8}$ University of Manchester, Manchester Academic Health Science Centre, Centre for Health Informatics, Vaughan House, Portsmouth St, Manchester, M13 9GB, UK

$22{ }^{9}$ Department Cardiothoracic Surgery, James Cook University Hospital, Middlesbrough, TS4 3BW, UK

24 * Corresponding Author: Mr. Joel Dunning, Address: Department Cardiothoracic Surgery, James Cook University Hospital, Middlesbrough, TS4 3BW, UK, Email:

26 joeldunning@ doctors.org.uk, Tel: +44 (0)1642 854612

Word count: 2979 (34 references)

28 Number of tables: 0 ; Number of figures: 5

Number of supplementary files: 1 


\section{ABSTRACT}

32 Context: Post-market evidence generation for medical devices is important yet limited for prosthetic aortic valve devices in the United Kingdom (UK).

34 Objective: To identify prosthetic aortic valve models that display unexpected patterns of mortality or re-intervention using routinely collected national registry data and record linkage.

36 Design: Observational study using the UK National Adult Cardiac Surgery Audit (NACSA) registry for procedures performed between 1998 and 2013. Valves were classified into series of

38 related models. Outcome tracking was performed using multifaceted record linkage. The median follow-up was 4.1 years (maximum 15.3 years). Cox proportional hazards regression with

40 random effects (frailty models) were used to model valve effects on the outcomes, with and without adjustment for (pre-)operative covariates.

42 Setting: All National Health Service and private hospitals in England and Wales who submit data to the NACSA registry.

44 Patients and Interventions: All patients undergoing first-time elective and urgent aortic valve replacement surgery ( \pm coronary artery bypass graft) with a mechanical ( $n=10$ series) or

46 biological ( $n=15$ series) prosthetic valve from 5 primary suppliers, and satisfying pre-specified data quality criteria were included ( $n=43,782$ biological, $n=11,084$ mechanical).

48 Main Outcome Measures: Time to all-cause mortality or aortic valve re-intervention (surgical or trans-catheter). There were 13,104 deaths and 723 re-interventions during follow-up.

50 Results: Two series of valves were associated with significantly increased hazard of death or reintervention were identified: Sorin Biological Series (frailty 1.18 [95\%PI: 1.06 to 1.32]) and 
52 Sorin Mitroflow series (frailty 1.19 [95\%PI: 1.09 to 1.31]). These results were robust to covariate adjustment, and sensitivity analyses. Three biological valve series were associated with

54 significantly decreased hazard.

Conclusions: Meaningful evidence from the analysis of routinely-collected registry data can

56 inform post-market surveillance of medical devices. Although the findings are associated with a number of caveats, two specific biological aortic valve series identified in this study may warrant

58 further investigation.

Word count (abstract): 300

60 Keywords: Post-market surveillance; prosthetic valves; aortic valves; survival; clinical registry data

62 


\section{INTRODUCTION}

In recent years there has been a shift in emphasis from establishing device safety and effectiveness before marketing, to post-market evidence generation and surveillence. ${ }^{1}$ The

66 United States Food and Drug Administration (US FDA) system for post-market surveillance has been found to be in need of strengthening. ${ }^{2,3}$ This need for improved surveillance systems was

68 recently highlighted by the international health scare caused by Poly Implant Prothèse (PIP) breast implants. ${ }^{4}$ Post-market surveillance systems have historically been reactive rather than

70 proactive in the United Kingdom (UK) as evidenced by concerns over hip prostheses leading to the UK National Joint Registry being established. ${ }^{5}$

Prosthetic heart valves have evolved significantly since the first valve replacement was performed in 1952. Although there are two main groups of prosthetic heart valve, tissue or

74 mechanical, there are a variety of different valves within these groups. There is a large body of literature on the long-term reliability of prosthetic heart valves, but these studies, whether randomised trials, ${ }^{6}$ observational, ${ }^{7}$ or case-series, ${ }^{8}$ typically compare a very small number of valves. Data from systematic benchmarking of long-term performance is not readily available. Government's Department of Health and the Society for Cardiothoracic Surgery in Great Britain

80 and Ireland (SCTS). ${ }^{9,10}$ Within 10-years a minimum dataset of clinical variables about heart valve replacement procedures had been entered for more than 45,000 patients. ${ }^{11}$ The UKHVR

82 fulfilled an important role: the ability to monitor trends in outcomes by different prosthetic valve models. It was setup to do this by recording valve model and serial numbers for implanted

84 prosthetic valves, and also by linkage to mortality data, including cause of death, from the Office 
for National Statistics (ONS). In 2004, funding was withdrawn due to cost and governance

86 issues, with its functionality partly subsumed by a national adult cardiac surgery register. ${ }^{12}$ Currently, the UK agency responsible for ensuring that medical devices meet applicable 88 standards of safety - the Medicines and Healthcare products Regulatory Agency (MHRA) collects data on acute valve failures submitted by healthcare professionals; however, in the

90 absence of a device-specific registry, the opportunity to detect patterns of unexpected outcomes are limited.

92 Prospective surveillance based on clinical registries that record device-specific information can identify important signals that passive reporting mechanisms may miss, ${ }^{13,14}$ and

94 there have been calls to move from reactive to proactive monitoring. ${ }^{14}$ As a prelude to any prospective surveillance programme, we present results for a retrospective cross-sectional

96 surveillance analysis of prosthetic valves implanted into patients undergoing aortic valve replacement (AVR) surgery with or without concomitant coronary artery bypass grafting $98(\mathrm{CABG})$ in England and Wales over the past 15-years. 


\section{Extraction and preprocessing of aortic valve surgery data}

A complete extract from the National Adult Cardiac Surgery Audit (NACSA) registry version 4.1.2), which is run by the National Institute for Cardiovascular Outcomes Research

104 (NICOR; an institute of University College London), was performed on 10th October 2014. This extract included all adult cardiac surgery procedures performed in UK National Health Service

106 (NHS) hospitals, some private hospitals and some hospitals in the Republic of Ireland. Case ascertainment of NHS procedure is expected to be high for most of the study period. ${ }^{12}$ As part of

108 a wider clinical epidemiological research and quality improvement programme, a regularly updated suite of 'data cleaning' rules developed by specialist clinicians were coded and applied

110 to the raw data (excluding the valve model data) prior to any analysis as summarized in the Appendix..$^{15,16}$

The initial filtering step was to extract all records corresponding to aortic valve surgery performed in hospitals located in England and Wales between 1st April 1998 and 31st March

114 2013. Data for one private hospital were removed prior to analysis pending local validation, as were all data for patients who had more than one record in the registry for the same admission 116 spell. For the purposes of this study, we selected all patients who underwent an AVR \pm CABG. We then excluded all records corresponding to: 1) patients having previous cardiac surgery; 2)

118 suspected incorrectly entered trans-catheter aortic valve implantation (TAVI) procedures (as identified using a rules-based approach); 3) emergency or salvage procedures; 4) unidentifiable

120 responsible consultant surgeon (as identified by a unique surgeon's General Medical Council number in the registry); 5) missing primary outcome data. 


\section{Record linkage}

To facilitate long-term monitoring of patient and valve status, we performed multiple

124 record linkages for each patient for life status, surgical reoperation, and TAVI as described in the Appendix.

\section{Valve model data and data quality}

Prostheses are recorded in the NACSA registry in two separate free-text fields: valve

128 name and valve model. There was inconsistency on how each hospital entered these data. An updated suite of data-processing scripts was written to map each recorded name and/or model to

130 a homogenous list of known prosthetic valves using a variety of information sources as described in the Appendix. For each record, we attempted to record the valve manufacturer, model, series,

132 and type (mechanical or biological, and xenograft type in the case of biological valves). Here, 'series' refers to a group of valve models from a single manufacturer considered related (See

134 Table S1 for groupings used). Not all valves could be accurately classified. When valve series was not clear, a subjective decision was made based on expert clinical opinion. Note that

136 manufacturer classification only reflects ownership as of 2015 to the best of our knowledge. Some models have been acquired by manufacturers through business mergers and acquisitions,

138 but are grouped together according to model.

Records which were irrelevant or featured gross inconsistencies were excluded, including

140 records that could either not be matched or which were matched to more than one manufacturer, series, or type, or which were matched to $>1$ model were excluded. Homografts, autografts,

142 rings, valve conduits, two particular model types, off-label procedures, and valves not produced by one of the UK primary suppliers were also excluded (see Appendix for details). The 5 
144 manufacturers included are Edwards Lifesciences (Irvine, CA, USA), Medtronic Inc. (Minneapolis, MN, USA), Sorin Group (Milan, Italy), St Jude Medical, Inc. (St. Paul, MN,

146 USA), and Vascutek Ltd. (a Terumo Company, Inchinnan, Scotland, UK).

\section{Study variables}

For each procedure, data were extracted for administrative factors, patient characteristics, comorbidities, surgical team, intra-operative factors, and post-operative outcomes. There were

150 few missing clinical data (all $>95 \%$ complete with the exception of the dichotomous creatinine variable [5.6\% missing], critical preoperative state $[7.4 \%$ missing], haemodynamics $[5.1 \%$

152 missing] and aortic valve pathology [10.1\% missing]). Details of study variable definitions and missing data imputation are given in the Appendix.

\section{Study outcomes}

The outcome for this study was time from surgery to the first event of death or re-

156 intervention. Patients were censored at the last follow-up time if alive and re-intervention free. Patients who died in-hospital on the day of surgery were recorded as having a nominal survival 158 time of 0.5 days. Follow-up data, until the point of discharge, were collected by the NACSA registry and post-discharge survival data were collected by record linkage to the ONS death

160 registry. Re-intervention was defined as surgical reoperation on the aortic valve for any reason or TAVI. Time-to-re-intervention data was collected by intra- and inter-record linkage as described 162 above.

\section{Statistical analysis}



indication. Valves were compared only at series-level. The Kaplan-Meier estimator was used to construct survival curves for the time-to-event outcome, and compared between valves using log-rank tests. Multivariable Cox proportional hazards regression models were used to adjust for potential differences with zero-mean valve series-level normally distributed random effects. The exponentiated random-effects - also known as the shared frailties — act multiplicatively on the

170 baseline hazard rate and therefore have an intuitive translation: frailty terms $>1$ correspond to increased hazard for a valve, and those < 1 correspond to decreased hazard. Frailties where the

172 corresponding $95 \%$ prediction interval lower limit lies above 1 indicate a valve with a significantly large hazard rate for the outcome. The focus of this study was not the identification

174 of prognostic factors, hence we limit reporting to the frailty effects. For comparison, unadjusted frailties are also reported. All analyses and data cleaning were performed in R (Version 3.3.1; R

176 Foundation for Statistical Computing, Vienna, Austria; http://www.R-project.org/). More detailed description of the statistical analysis is given in the Appendix. A number of different

178 sensitivity analyses were performed (reported in the Appendix). All inferences remained broadly consistent. 


\section{RESULTS}

From 79,345 AVR \pm CABG records with a biological or mechanical prosthesis, a total of 54,866 records were retained for analysis (Figure 1; Appendix), from 37 hospitals (including 4 184 private units) and 344 consultant surgeons.

Table S1 lists the valves included, which were grouped into 15 and 10 series of

186 biological and mechanical valves, respectively. Figure 2 shows an increasing trend in the implantation rate of biological valves during the study period, stabilising at $86 \%$. Figure 3 shows the number of valves implanted by time for each series. The distribution of patient age at surgery (Figure 4) indicates homogeneity between the valve-series within type (biological and

190 mechanical), with the exception of greater patient ages for the Medtronic Hall series, Vascutek Ultracor series, Edwards Lifesciences Mechanical series, and Sorin Sutureless series relative to

192 others of the same type. Plots for logistic EuroSCORE, gender, native valve pathology, procedure, BMI, valve size, and NYHA grade are shown in Figures S1-S8.

194 Valve outcomes

During a median follow-up of 4.1 years (maximum follow-up 15.3-years), 13,104 deaths

196 (11,353 biological; 1751 mechanical) were recorded and there were 723 (571 biological; 152 mechanical) re-interventions, (682 were surgical procedures and 41 were TAVIs). Results from 198 the Kaplan-Meier estimator analysis and pathological data for surgical re-interventions are described in the Appendix.

After adjustment, the random effects survival model indicated that the Sorin Mitroflow series (frailty 1.19 [95\%PI: 1.09 to 1.31]) and Sorin Biological series (frailty 1.18 [95\%PI: 1.06 202 to 1.32]) displayed larger hazard than expected (Figure 5). To place the outcomes into 
perspective, the 10-year overall freedom from re-intervention or death rates for the 2 valves were

$20433.8 \%$ [95\%CI: $31.3 \%$ to $36.5 \%$ ] and $41.4 \%$ [95\%CI: $37.6 \%$ to $45.6 \%$ ], respectively, compared to the overall average of $47.2 \%$ [ $95 \%$ CI: $46.2 \%$ to $48.1 \%$ ] for all non-Sorin biological valves.

206 Although non-significant, the lower 95\% PI for the Medtronic ATS-3f series only marginally crossed the line of unity (frailty 1.21 [95\%PI: 1.00 to 1.47$]$ ). For mechanical valves, the

208 Medtronic Hall valve had a significantly larger unadjusted hazard (unadjusted frailty 1.48 [95\%PI: 1.22 to 1.80$]$ ). However, after adjustment this was considerably shrunken (adjusted

210 frailty 1.10 [95\%PI: 0.97 to 1.24$]$ ), reflecting the greater patient age relative to the profile of other mechanical valves. Additional results are provided in the Appendix

212 There were three prosthetic valves with a significant reduction in hazard (Figure 5): the Edwards Lifesciences Perimount series (frailty 0.88 [95\%PI: 0.80 to 0.96$]$ ), the Edwards

214 Lifesciences Perimount Magna series (frailty 0.88 [95\%PI: 0.80 to 0.96 ]), and the Medtronic Hancock series (frailty 0.88 [95\%PI: 0.78 to 0.98$]$ ).

216 A subgroup analysis of all bioprosthesis records performed on or after 1st April 2008 $(n=23,834)$ showed that the lower $95 \%$ prediction interval limit was $<1$ for every valve after 218 adjustment (see Appendix). 
We analysed a comprehensive clinical registry to measure re-intervention-free survival in

222 a large series of patients undergoing AVR in the UK. Two series of prosthetic aortic valves were associated with significantly increased hazards of death or re-intervention, relative to the

224 population of prosthetic valves implanted in England and Wales from large suppliers. Similarly, three series of prosthetic valves were associated with decreased hazards. Inferences remained

226 broadly consistent following covariate adjustment and sensitivity analyses. This study has shown that routinely-collected clinical registry data can be exploited, in conjunction with multifaceted

228 record linkage, to perform long-term device surveillance.

There is a large literature examining outcomes following different prosthetic AVR

230 implants. Few studies, however, reflect national data. Moreover, the evidence-base is mixed. For example, some studies have suggested an inferior performance of the Sorin Mitroflow ${ }^{17,18}$

232 whereas on the other hand, others have demonstrated long-term durability and haemodynamic performance. ${ }^{19,20}$

The NHS number-a unique patient identifier — enables record linkage across clinical registries and other data sources. It would be feasible to exploit this to link across further data

236 sources (e.g. trace readmission from administrative data). In fact, strategic linking of complementary registries and data sources is a "foundational architectural construct"

238 recommendation of the US Medical Device Registries Task Force. ${ }^{21}$ Furthermore, record-linkage could be further extended using unique serial numbers of implanted devices (including prosthetic

240 aortic valves) to device manufacturer databases to improve ongoing research, augment clinical trial follow-up after completion, and to allow traceability in case of serious fault detection. The 
242 planned role out by the US FDA of a unique device identification system integrated for use with electronic health records would allow scalable cross-speciality surveillance. ${ }^{22}$

244 We explored outcomes in prosthetic valves cross-sectionally using 15-years of data. Moving forward, this is not a suitable approach for post-market device surveillance, which 246 should be dynamic, providing regular updates, to achieve superiority over existing passive reporting mechanisms. It is conceivable that signals of unexpected patterns of outcomes could 248 have been detected earlier on. The Data Extraction and Longitudinal Trend Analysis (DELTA) network study is a validated example of such a tool, which has utilised propensity score

250 matching and statistical process control methodology to evaluate the safety of high-risk cardiovascular devices for perioperative binary outcomes. ${ }^{13,21,23,24}$ Similar efforts for post-market

252 surveillance of pharmacological products are also on-going. ${ }^{25}$ Whilst the methodology applied here was relatively simplistic, what we have demonstrated is that routinely collected clinical

254 registry data can be leveraged for evaluating performance of medical devices, even when this was not a primary goal of the data collection programme. With some improvements to the data 256 collection mechanisms, this messy real-world registry, or other registries, data could be analysed using alternative platforms. 


\section{LIMITATIONS}

\section{Data quality}

Research with routinely-collected healthcare data inevitably raises questions over data

262 quality. Many of the data on clinical variables are of high quality, owing to the fact they are used for national governance. ${ }^{16,26}$ Valve-specific data, on the other hand, are not subject to similar

264 quality management. As the valve model data were collected as free-text inputs, more data quality issues were present than for equivalent clinical information collected using structured

266 inputs. Data quality is expected to improve in the future, due to increased scrutiny of device monitoring. Caution must therefore be taken when interpreting the results, as there is potential

268 for coding errors by the surgeon.

\section{Valve classification}

Focusing surveillance on a coarsened valve grouping — series — as opposed to valve models ensured that the maximum number of records would be available for analysis. This

272 decision, whilst allowing us to retain more records for analysis, introduces limitations. Firstly, different models in a series, including stented and stentless models, or different generations of

274 the same model, might have a variable effect on outcome. For example, the latest generations of Sorin Mitroflow valves are processed with a phospholipid reduction treatment to mitigate

276 calcification. This might lead to improved performance compared to earlier generations.

Secondly, not all valve series are clearly delineated due to either historical device company

278 purchases/mergers or naming conventions. Similarity in naming means that valves identified to the series level but not the model level might potentially be misclassified. This is discussed 280 further in the Appendix. 


\section{Covariate adjustment}

The adjustment data used in this study derives from a national clinical registry, which is widely accepted to be superior to administrative data. ${ }^{27}$ There was no $a$ priori expectation of

284 gross selection bias by valve series within valve type, nor was substantial heterogeneity observed, unlike in some other post-market surveillance studies for cardiovascular devices. ${ }^{13}$

286 However, there has been a shift in patient risk profiles over time, ${ }^{28}$ which might confound with market availability of certain valves. We adjusted for baseline risk factors, as well for a number

288 of clinical valve-related variables, and contrasted the change in inference with that of the unadjusted model. Another potential source of bias stems from the missing data being imputed

290 according to a (gender-stratified) mean/mode approach $;{ }^{29}$ however, missing data was not considered substantial. One should also note that the number of random-effects was quite small

292 for a frailty model. Additionally, no adjustment for institutional effects were included, which could conflate with models implanted.

\section{Study outcomes}

In some records, patient ID was missing, which can reduce the ability to track patients.

296 Moreover, tracking was terminated at different time points for different endpoints: December2012 for TAVI, March-2013 for surgical re-intervention, and July-2013 for survival. Since the

298 focus of the study was on valve surveillance, rather than patient outcomes monitoring, we only analysed the time to first event. We also note that sample sizes differed substantially between

300 models. This was due to multiple factors, including market availability; some are relatively new and others have been withdrawn, which might impact on the ability to detect valves that have 302 significantly different event hazards. ${ }^{30}$ Some newer implanted valves may not yet have sufficient 
volume to show significantly different outcomes. We have also defined a composite outcome for 304 analysis, rather than analysing death and re-intervention as a competing risk. ${ }^{31}$ Differences in outcomes may be attributable to different causes; for example, if a valve migrates it will lead to 306 an increase in re-operation, as was observed with the $3 \mathrm{f}$ Enable Aortic Bioprosthesis. ${ }^{32}$

The greatest clinical limitations of this study are its relatively short follow-up and lack of 308 other clinical outcomes. ${ }^{33}$ The median follow-up time was 4.1-years, however valve failure is most likely to occur later on, especially in the context of mechanical valves. In fact, only 152

310 surgical re-interventions were observed in the mechanical valve group. Finally, we excluded patients who had multiple surgical records within a single admission; however, there were only 31234 such cases satisfying the inclusion criteria for the study. 


\section{CONCLUSIONS}

The need for such post-marketing surveillance of medical devices was made clear by the

316 PIP breast implant and other medical device scares, ${ }^{3}$ yet infrastructure is lacking. We have shown that a national clinical registry, linked to other routinely-collected data, might be used to

318 inform post-market surveillance programmes. By analysing 15-years of data on AVR procedures in England and Wales we identified 2 prosthetic valves that may warrant further

320 scrutiny through additional studies. As Taylor noted about valve monitoring nearly 3-decades ago, "overreaction is as inappropriate as complacency". ${ }^{34}$ Given the limitations of the study, the

322 signals shown here should only serve as a hypothesis generating, and not be misinterpreted as causal effects. 


\section{STUDY APPROVAL}

This study was approved by the NICOR NACSA Research Board (study reference 13ACS-09), and the need to obtain informed consent from patients was waived as patient

328 identifiable information was either removed or pseudonymised.

\section{ACKNOWLEDGEMENTS}

The authors acknowledge all members of the Society for Cardiothoracic Surgery in Great Britain and Ireland who contribute data to the SCTS database. The National UK TAVI Registry

332 steering group agreed to the use of record linkage for the purposes of tracking valve-in-valve reinterventions. The National Institute for Cardiovascular Outcomes Research (NICOR),

334 University College London (UCL), London, provided the data for this study. The authors also thank Miss Rebecca Cosgriff (former NACSA project manager for NICOR) for obtaining the 336 hospital code lists; Mr John McKenna (formerly of Vascutek Terumo) for helping to identify some of the valves; and representatives of the valve companies for their helpful support. No one 338 acknowledged here received or provided compensation for their services.

\section{FUNDING}


BB has received honoraria from Edwards Lifesciences in the last 3-years. JD1 (J.

Dunning) has received proctoring fees from Cardica. MD-H has received honoraria from St. Jude

348 Medical, Edwards Lifesciences and Maquet, and research grants from Edwards Lifesciences and

St. Jude Edwards. NM has received fees for consultancy and proctoring from Medtronic,

350 consultancy for Tendyne (Direct Flow), and speaking from Abbott. All other authors have no conflicts of interests to declare.

\section{CONTRIBUTORSHIP STATEMENT}

JD1 (J. Dunning) conceived the idea for the study. All co-authors contributed to the

354 development of the study analysis plan. GLH and SWG cleaned the clinical surgical data. GLH, BB, AJB, MD-H and JD1 cleaned the valve model data. GLH performed the data linkage and 356 statistical analysis. GLH, JD1, BB, SWG, JP and JD2 (J. Deanfield) wrote the manuscript, which was critically reviewed for intellectual content by all authors. All authors have read and agree to 358 the final version. JD1 acts as guarantor for the manuscript.

\section{DATA SHARING}

The United Kingdom National Adult Cardiac Surgery Audit registry and the United Kingdom National TAVI Audit Registry are available to researchers upon application to the

362 National Institute of Cardiovascular Outcomes Research (NICOR), University College London. Full details on the NICOR data sharing application process are available at

364 https://www.ucl.ac.uk/nicor/access/application [last accessed: 16th October 2015]. 


\section{REFERENCES}

1. Rathi VK, Krumholz HM, Masoudi FA, Ross JS. Characteristics of clinical studies conducted over the total product life cycle of high-risk therapeutic medical devices receiving FDA premarket approval in 2010 and 2011. JAMA 2015;314(6):604-12.

2. Ross JS. Strengthening medical device postmarket safety surveillance. JAMA Intern. Med. 2015;175(8):1350-1.

372 3. Hauser RG. Here we go again--failure of postmarketing device surveillance. N. Engl. J. Med. 2012;366(10):873-5.

374 4. Maijers MC, Niessen FB. Prevalence of rupture in poly implant Prothèse silicone breast implants, recalled from the European market in 2010. Plast. Reconstr. Surg. 2012;129(6):1372-8.

5. Smith AJ, Dieppe P, Vernon K, Porter M, Blom AW. Failure rates of stemmed metal-on-

6. Dalmau MJ, González-Santos JM, Blázquez JA, et al. Hemodynamic performance of the Medtronic Mosaic and Perimount Magna aortic bioprostheses: five-year results of a prospectively randomized study. Eur. J. Cardio-Thoracic Surg. 2011;39(6):844-852.

7. Jamieson WRE, David TE, Feindel CMS, Miyagishima RT, Germann E. Performance of the Carpentier-Edwards SAV and Hancock-II porcine bioprostheses in aortic valve replacement. J. Heart Valve Dis. 2002;11(3):424-30.

8. David TE, Armstrong S, Maganti M. Hancock II bioprosthesis for aortic valve replacement: the gold standard of bioprosthetic valves durability? Ann. Thorac. Surg. 2010;90(3):775-81.

9. Taylor KM, Gray SA, Livingstone S, Brannan JJ. The United Kingdom heart valve registry. J. Heart Valve Dis. 1992;1(2):152-9.

10. Keogh BE, Kinsman R. The Society for Cardiothoracic Surgery in Great Britain \& Ireland: The Fifth National Adult Cardiac Surgical Database Report. Henley-on-Thames, UK: Dendrite Clinical Systems Ltd; 2003. 
394 11. Taylor KM. The United Kingdom Heart Valve Registry: the first 10 years. Heart 1997;77:295-296.

396 12. Bridgewater B. Cardiac registers: The adult cardiac surgery register. Heart 2010;96(18):1441-3. doi:10.1136/hrt.2010.194019.

398 13. Kumar A, Matheny ME, Ho KKL, et al. The data extraction and longitudinal trend analysis network study of distributed automated postmarket cardiovascular device safety surveillance. Circ. Cardiovasc. Qual. Outcomes 2015;8(1):38-46.

14. Rumsfeld JS, Peterson ED. Achieving meaningful device surveillance: from reaction to proaction. JAMA 2010;304(18):2065-6.

15. Hickey GL, Grant SW, Cosgriff R, et al. Clinical registries: governance, management, analysis and applications. Eur. J. Cardio-Thoracic Surg. 2013;44(4):605-14.

16. Hickey GL, Cosgriff R, Grant SW, et al. A technical review of the United Kingdom 406 National Adult Cardiac Surgery Governance Analysis 2008-11. Eur. J. Cardio-Thoracic Surg. 2014;45(2):225-33.

408 17. Alvarez JR, Sierra J, Vega M, et al. Early calcification of the aortic Mitroflow pericardial bioprosthesis in the elderly. Interact. Cardiovasc. Thorac. Surg. 2009;9(5):842-6.

410 18. Butany J, Feng T, Luk A, Law K, Suri R, Nair V. Modes of failure in explanted mitroflow pericardial valves. Ann. Thorac. Surg. 2011;92(5):1621-7.

412 19. Yankah CA, Pasic M, Musci M, et al. Aortic valve replacement with the Mitroflow pericardial bioprosthesis: durability results up to 21 years. J. Thorac. Cardiovasc. Surg. $414 \quad$ 2008;136(3):688-96.

20. ISTHMUS Investigators. The Italian study on the Mitroflow postoperative results (ISTHMUS): a 20-year, multicentre evaluation of Mitroflow pericardial bioprosthesis. Eur. J. Cardio-Thoracic Surg. 2011;39(1):18-26; discussion 26.

418 21. Krucoff MW, Sedrakyan A, Normand S-LT. Bridging unmet medical device ecosystem needs with strategically coordinated registries networks. JAMA 2015.

420 22. Rising J, Moscovitch B. The Food and Drug Administration's unique device identification 
system: better postmarket data on the safety and effectiveness of medical devices. JAMA Intern. Med. 2014;174(11):1719-20.

23. Resnic FS, Donnelly S, Normand S-LT, Matheny ME. Automated surveillance to detect postprocedure safety signals of approved cardiovascular devices. J. Am. Med. Assoc. 2010;304(18):2019-2027.

426 24. Vidi VD, Matheny ME, Donnelly S, Resnic FS. An evaluation of a distributed medical device safety surveillance system: The DELTA network study. Contemp. Clin. Trials 2011;32(3):309-317.

25. Moore TJ, Furberg CD. Electronic health data for postmarket surveillance: a vision not realized. Drug Saf. 2015;38(7):601-610.

26. Bridgewater B, Hickey GL, Cooper G, Deanfield J, Roxburgh JC. Publishing cardiac surgery mortality rates: lessons for other specialties. BMJ 2013;346:f1139.

27. Shahian DM, Edwards FH, Jacobs JP, et al. Public reporting of cardiac surgery performance: part 2 - implementation. Ann. Thorac. Surg. 2011;92(3 Suppl):S12-23. doi:10.1016/j.athoracsur.2011.06.101.

28. Hickey GL, Grant SW, Murphy GJ, et al. Dynamic trends in cardiac surgery: why the logistic EuroSCORE is no longer suitable for contemporary cardiac surgery and implications for future risk models. Eur. J. Cardio-Thoracic Surg. 2013;43(6):1146-52.

29. Horton NJ, Kleinman KP. Much ado about nothing: A comparison of missing data 440 methods and software to fit incomplete data regression models. Am. Stat. 2007;61(1):7990 .

442 30. Hernán MA. The hazards of hazard ratios. Epidemiology 2010;21(1):13-15. doi:10.1016/j.cmet.2012.08.002.

444 31. Grunkemeier GL, Jin R, Eijkemans MJC, Takkenberg JJM. Actual and actuarial probabilities of competing risks: apples and lemons. Ann. Thorac. Surg. 2007;83(5):15861592.

32. Medtronic. Update to urgent field safety notice. 2015. Available at: https://www.swissmedic.ch/recalllists_d1/11658/Vk_20141120_13_en_Update.pdf. 
Accessed October 29, 2015.

450 33. Akins CW, Miller DC, Turina MI, et al. Guidelines for reporting mortality and morbidity after cardiac valve interventions. Eur. J. Cardio-Thoracic Surg. 2008;33(4):523-528.

452 34. Taylor KM. Acute failure of artificial heart valves. BMJ 1988;297(6661):1473-1474.

454 


\section{FIGURE LEGENDS}

456 Figure 1. Flowchart of study data.

Figure 2. Trend in proportion of biological and mechanical valves implanted over the study 458 period.

Figure 3. Trends in number of valves implanted by valve series over the study period.

460 Figure 4. Box-and-whisker plots of patient age at time of surgery stratified by valve series.

Figure 5. Frailty effects (black filled circles) and 95\% prediction intervals (black lines) by valve 462 series for time-to-death and time-to-re-intervention as calculated for Cox random effects models (with and without adjustment for other patient and operative risk factors). Red dashed line 464 denotes 'no effect'. 